\title{
Erratum: One-loop correction to heavy dark matter annihilation [Phys. Rev. D 95, 055001 (2017)]
}

\author{
Grigory Ovanesyan, Nicholas L. Rodd, Tracy R. Slatyer, and Iain W. Stewart
}

(Received 11 November 2019; published 3 December 2019)

DOI: $10.1103 /$ PhysRevD.100.119901

In Sec. III, Eq. (13) should read

$$
\begin{aligned}
& C_{1}(\mu)=-\frac{\pi \alpha_{2}(\mu)}{m_{\chi}}+\frac{\alpha_{2}(\mu)^{2}}{4 m_{\chi}}\left[2 \ln ^{2} \frac{\mu^{2}}{4 m_{\chi}^{2}}+2 \ln \frac{\mu^{2}}{4 m_{\chi}^{2}}+2 i \pi \ln \frac{\mu^{2}}{4 m_{\chi}^{2}}+12-\frac{11 \pi^{2}}{6}\right], \\
& C_{2}(\mu)=\frac{\pi \alpha_{2}(\mu)}{m_{\chi}}-\frac{\alpha_{2}(\mu)^{2}}{2 m_{\chi}}\left[\ln ^{2} \frac{\mu^{2}}{4 m_{\chi}^{2}}+3 \ln \frac{\mu^{2}}{4 m_{\chi}^{2}}-i \pi \ln \frac{\mu^{2}}{4 m_{\chi}^{2}}+2-\frac{5 \pi^{2}}{12}\right] .
\end{aligned}
$$

In detail the +12 and +2 in these equations were corrected from +8 and 0 .

These changes result from corrections to the counter-terms and threshold corrections, which appeared in Appendix A, and affected results in our original Eqs. (A32), (A33), (A36), (A39), (A40), and (A41). For simplicity we repeat that entire part of the appendix below with the corrected equations, replacing the material between Eqs. (A31) and (A42) of the original paper.

\section{Counter-terms}

To begin with, as $B_{3}$ vanishes at tree level there are no counter-term corrections to its value at one loop. Instead we only need to consider graphs that would contribute to $B_{1}$ and $B_{2}$, of which there are three:

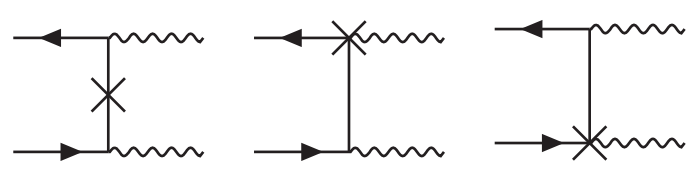

The graph on the left corresponds to the internal wave-function and mass renormalization of the DM-renormalization factors denoted as $Z_{\chi}$ and $Z_{m}$-whilst the remaining two graphs account for the renormalization of the DM and electroweak gauge boson interaction vertex $g_{2} \bar{\chi} W \chi$ - here $Z_{1}$ (which includes coupling and external line wave function renormalization). Now if we calculate the above three graphs, we find a contribution proportional to the tree-level amplitude $\mathcal{M}_{\text {tree }}$, as well as a term that would contribute to $B_{3}$. The contribution to $B_{3}$ is canceled by the additional $s$-channel type counter-term graphs not drawn, so the full counter-term contribution leaves only:

$$
\left(2 \delta_{1}-\delta_{\chi}-\delta_{m}\right) \mathcal{M}_{\text {tree }}
$$

where we have used $Z_{i}=1+\delta_{i}$.

Next, when determining the $\delta_{i}$ we need to pick a scheme. As explained above, when calculating matching coefficients it is easiest to work in the on-shell scheme for wave function renormalization to ensure we do not have to worry about residues from the LSZ reduction. The meaning of the on-shell values of $\delta_{\chi}$ and $\delta_{m}$ is clear, whereas for $\delta_{1}$ we must write this out more explicitly. By definition we know $\delta_{1}=\delta_{g_{2}}+\frac{1}{2} \delta_{W}+\delta_{\chi}$, where $\delta_{g_{2}}$ and $\delta_{W}$ are the counter-terms for the coupling and gauge boson wave functions, respectively. For the gauge boson wave function we use the on-shell scheme as usual. For the coupling counter-term, however, we define it in the $\overline{\mathrm{MS}}$ scheme. Since our full theory is defined with the DM as a propagating degree of freedom, this coupling is defined above the $m_{\chi}$. In the EFT the DM is integrated out, so the appropriate coupling for the matching is one defined below $m_{\chi}$. We put this issue aside for now and return to it in the next section. 
The above choices then define our scheme for $\delta_{1}$ in a manner that ensures all residues are still 1 . With this scheme, we can then calculate the relevant counter-terms for Majorana DM and find ${ }^{1}$ :

$$
\begin{aligned}
& \delta_{\chi}=-\frac{\alpha_{2}}{4 \pi}\left[\frac{2}{\epsilon_{\mathrm{UV}}}+\frac{4}{\epsilon_{\mathrm{IR}}}+12 L+12 \ln 2+8\right], \\
& \delta_{m}=-\frac{\alpha_{2}}{4 \pi}\left[\frac{6}{\epsilon_{\mathrm{UV}}}+12 L+12 \ln 2+8\right], \\
& \delta_{W}=-\frac{\alpha_{2}}{4 \pi}\left[\frac{2 n_{D}-11}{6 \epsilon_{\mathrm{UV}}}+\frac{19-2 n_{D}}{6 \epsilon_{\mathrm{IR}}}+\frac{8}{3} L+\frac{8}{3} \ln 2\right], \\
& \delta_{g_{2}}=-\frac{\alpha_{2}}{4 \pi}\left[\frac{35-2 n_{D}}{12 \epsilon_{\mathrm{UV}}}\right], \\
& \delta_{1}=-\frac{\alpha_{2}}{4 \pi}\left[\frac{4}{\epsilon_{\mathrm{UV}}}+\frac{67-2 n_{D}}{12 \epsilon_{\mathrm{IR}}}+\frac{40}{3} L+\frac{40}{3} \ln 2+8\right],
\end{aligned}
$$

where $n_{D}$ is again the number of left-handed SM doublets. Recall that in determining the counter-terms we cannot neglect scaleless integrals as we did for the main calculation, so their contribution has been included here, and we explicitly distinguish $\epsilon_{\mathrm{UV}}$ from $\epsilon_{\mathrm{IR}}$. Substituting these results into Eq. (A31), we find the crossed contribution is:

$$
\begin{aligned}
& B_{1}^{[\mathrm{CT}]}=\frac{\alpha_{2}}{4 \pi}\left[\frac{2 n_{D}-43}{6 \epsilon_{\mathrm{IR}}}-\frac{8}{3} L-\frac{8}{3} \ln 2\right], \\
& B_{2}^{[\mathrm{CT}]}=-\frac{1}{2} B_{1}^{[\mathrm{CT}]}, \\
& B_{3}^{[\mathrm{CT}]}=0 .
\end{aligned}
$$

Interestingly the counter-term contribution is UV finite. This implies that the sum of all one-loop graphs before adding in counter-terms must be UV finite. Given that we used dimensional regularization to regulate both UV and IR divergences this cannot be immediately read off from our results, but going back to the integrals and keeping track of the UV divergences we confirmed that the sum is indeed UV finite.

Note if our DM field had instead been a Dirac fermion, the above result would vary, but in a way that is exactly canceled when we account for the threshold corrections in the coupling, as discussed in the next section. In detail, $\delta_{\chi}$ and $\delta_{m}$ remain unchanged, whereas we now have

$$
\begin{aligned}
& \delta_{W}=-\frac{\alpha_{2}}{4 \pi}\left[\frac{2 n_{D}-3}{6 \epsilon_{\mathrm{UV}}}+\frac{19-2 n_{D}}{6 \epsilon_{\mathrm{IR}}}+\frac{16}{3} L+\frac{16}{3} \ln 2\right], \\
& \delta_{g_{2}}=-\frac{\alpha_{2}}{4 \pi}\left[\frac{27-2 n_{D}}{12 \epsilon_{\mathrm{UV}}}\right], \\
& \delta_{1}=-\frac{\alpha_{2}}{4 \pi}\left[\frac{4}{\epsilon_{\mathrm{UV}}}+\frac{67-2 n_{D}}{12 \epsilon_{\mathrm{IR}}}+\frac{44}{3} L+\frac{44}{3} \ln 2+8\right],
\end{aligned}
$$

so that in the Dirac fermion case

$$
\begin{aligned}
& B_{1}^{[\mathrm{CT}]}=\frac{\alpha_{2}}{4 \pi}\left[\frac{2 n_{D}-43}{6 \epsilon_{\mathrm{IR}}}-\frac{16}{3} L-\frac{16}{3} \ln 2\right], \\
& B_{2}^{[\mathrm{CT}]}=-\frac{1}{2} B_{1}^{[\mathrm{CT}]}, \\
& B_{3}^{[\mathrm{CT}]}=0 .
\end{aligned}
$$

\footnotetext{
${ }^{1}$ As pointed out in [1], the original version of this paper had incorrect coefficients in some entries of these counter terms. This affected the constant terms appearing in $C_{1}$ and $C_{2}$ in Eq. (13), namely the +12 and +2 terms, respectively. The resulting changes to the figures are invisible to the eye. We thank the authors of that work for bringing this to our attention.
} 


\section{Threshold corrections in the coupling}

Throughout the above calculation we have treated the DM as a propagating degree of freedom and included its effects in loop diagrams. This implies that the coupling used so far above in this appendix implicitly depends on $n_{D}+1$ flavors ( $n_{D}$ left-handed SM doublets and one Majorana DM fermion); i.e., we have used $\alpha_{2}=\alpha_{2}^{\left(n_{D}+1\right)}(\mu)$. In the EFT however, the DM is no longer a propagating field and so the appropriate coupling is $\alpha_{2}^{\left(n_{D}\right)}(\mu)$. At order $\alpha_{2}^{2}$, which we are working to at one loop, the distinction will lead to a finite contribution because of the matching at the scale $\mu=m_{\chi}$, which we calculate in this section.

Let us start by reviewing the standard treatment of a running coupling in the $\overline{\mathrm{MS}}$ scheme. This running is captured by the $\beta$-function, which is defined by $\beta\left(\alpha_{2}\right)=\mu d \alpha_{2} / d \mu$, where here $\alpha_{2}$ is the renormalized coupling; the bare coupling is independent of $\mu$. The $\beta$-function can be written as:

$$
\beta\left(\alpha_{2}\right)=-2 \epsilon \alpha_{2}-\frac{b_{0}}{2 \pi} \alpha_{2}^{2}+\ldots,
$$

where we have expanded it to the order needed for this threshold matching analysis. At this order the LL solution for the running of the coupling is:

$$
\alpha_{2}(\mu)=\frac{\alpha_{2}\left(\mu_{0}\right)}{1+\alpha_{2}\left(\mu_{0}\right) \frac{b_{0}}{2 \pi} \ln \frac{\mu}{\mu_{0}}}
$$

In order to determine the threshold matching correction at the one-loop order we are working it suffices to simply demand that the coupling is continuous at the scale $m_{\chi}$, and this is captured by a difference in $b_{0}$. For our problem we define $b_{0}^{\left(n_{D}+1\right)}$ to be the value above $m_{\chi}$ and $b_{0}^{\left(n_{D}\right)}$ the value below. Then using Eq. (A37) to define $\alpha_{2}^{\left(n_{D}+1\right)}(\mu)$ and $\alpha_{2}^{\left(n_{D}\right)}(\mu)$, it suffices to demand they match at a scale $m_{\chi}$, which gives:

$$
\alpha_{2}^{\left(n_{D}+1\right)}(\mu)=\alpha_{2}^{\left(n_{D}\right)}(\mu)\left[1+\frac{\alpha_{2}^{\left(n_{D}\right)}(\mu)}{2 \pi}\left(b_{0}^{\left(n_{D}\right)}-b_{0}^{\left(n_{D}+1\right)}\right) \ln \frac{\mu}{m_{\chi}}+\ldots\right] .
$$

So now we just need to determine $b_{0}^{\left(n_{D}\right)}-b_{0}^{\left(n_{D}+1\right)}$. In general for a theory containing just gauge bosons, Weyl fermions (WF), Dirac fermions (DF), Majorana fermions (MF) and charged scalars (CS), we can write:

$$
b_{0}=\frac{11}{3} C_{A}-\frac{2}{3} \sum_{i \in \mathrm{WF}} C\left(R_{i}\right)-\frac{4}{3} \sum_{i \in \mathrm{DF}} C\left(R_{i}\right)-\frac{2}{3} \sum_{i \in \mathrm{MF}} C\left(R_{i}\right)-\frac{1}{3} \sum_{i \in \mathrm{CS}} C\left(R_{i}\right) .
$$

Our calculation can involve all of these ingredients: electroweak gauge bosons, the left-handed SM fermions (which are Weyl because only one chirality couples to the gauge bosons), the Majorana or Dirac DM fermion and the Higgs. Then using $C_{A}=2, C(R)=1 / 2$ for the SM left-handed fermions and the Higgs, and $C(R)=2$ for the adjoint Majorana Wino, we conclude:

$$
\begin{aligned}
b_{0}^{\left(n_{D}\right)} & =\frac{43-2 n_{D}}{6}, \\
b_{0}^{\left(n_{D}+1\right)} & =\frac{35-2 n_{D}}{6} .
\end{aligned}
$$

From this Eq. (A38) tells us that to the order we are working:

$$
\alpha_{2}^{\left(n_{D}+1\right)}(\mu)=\alpha_{2}^{\left(n_{D}\right)}(\mu)\left[1+\frac{\alpha_{2}^{\left(n_{D}\right)}(\mu)}{4 \pi}\left(\frac{8}{3} L+\frac{8}{3} \ln 2\right)\right] .
$$

Now as there is only a difference between the couplings at next to leading order, this only corrects the tree level result stated in Eq. (A4). As such the impact of changing to the coupling defined below $m_{\chi}$, which is relevant for the matching, is to add the following contribution: 


$$
\begin{aligned}
& B_{1}^{[\text {Matching }]}=\frac{\alpha_{2}}{4 \pi}\left[\frac{8}{3} L+\frac{8}{3} \ln 2\right], \\
& B_{2}^{[\text {Matching }]}=-\frac{1}{2} B_{1}^{[\text {Matching }]}, \\
& B_{3}^{[\text {Matching }]}=0
\end{aligned}
$$

where after adding this contribution now here and in all earlier one-loop results we can simply take $\alpha_{2}=\alpha_{2}^{\left(n_{D}\right)}$.

In the Dirac case, the wino contributes differently to $b_{0}^{\left(n_{D}+1\right)}$, giving

$$
b_{0}^{\left(n_{D}+1\right)}=\frac{27-2 n_{D}}{6},
$$

and accordingly we find

$$
\begin{aligned}
& B_{1}^{[\text {Matching }]}=\frac{\alpha_{2}}{4 \pi}\left[\frac{16}{3} L+\frac{16}{3} \ln 2\right], \\
& B_{2}^{[\text {Matching }]}=-\frac{1}{2} B_{1}^{[\text {Matching }]}, \\
& B_{3}^{[\text {Matching }]}=0 .
\end{aligned}
$$

Note that these corrections from threshold matching for the coupling exactly cancel the $L$ and $\ln 2$ terms that appear in $B_{1,2}^{[\mathrm{CT}]}$. As alluded to above, although the counter-term and threshold matching results individually differ for the Dirac or Majorana cases, when combined these terms lead to the same result for the final $B_{i}^{(1)}$ coefficients given in Eq. (A45) below.

\section{Combination}

Combining the 25 graphs above with the counter-terms and the matching contributions, we arrive at the following result:

$$
\begin{aligned}
& B_{1}^{(1)}=\frac{\alpha_{2}}{4 \pi}\left[-\frac{4}{\epsilon^{2}}-\frac{48 L+12 i \pi-55-2 n_{D}}{6 \epsilon}-8 L^{2}-4 L-4 i \pi L-12+\frac{11 \pi^{2}}{6}\right], \\
& B_{2}^{(1)}=\frac{\alpha_{2}}{4 \pi}\left[\frac{2}{\epsilon^{2}}+\frac{48 L-12 i \pi+79-2 n_{D}}{12 \epsilon}+4 L^{2}+6 L-2 i \pi L+2-\frac{5 \pi^{2}}{12}\right], \\
& B_{3}^{(1)}=\frac{\alpha_{2}}{4 \pi}\left[\frac{n_{D}-72 \ln 2-71+3 \pi^{2}}{12}\right],
\end{aligned}
$$

where recall $L=\ln \mu / 2 m_{\chi}, n_{D}$ is the number of SM left-handed doublets and now all $\epsilon=\epsilon_{\mathrm{IR}}$.

As explained in detail at the outset of the calculation, the one-loop contribution to the matching coefficient is just the finite part of this result. Combining this with the tree-level term in Eq. (A4) and mapping back to $C_{r}$ using Eq. (A3) then gives us the Wilson coefficients in Eq. (13), which we set out to justify.

If instead we had a Dirac DM triplet rather than a Majorana, then the only impact on the above would be for $B_{3}^{(1)}$, and we would instead have

$$
B_{3}^{(1)}=\frac{\alpha_{2}}{4 \pi}\left[\frac{n_{D}-72 \ln 2-43}{12}\right]
$$

[1] M. Beneke, A. Broggio, C. Hasner, and M. Vollmann, Phys. Lett. B 786, 347 (2018). 